\title{
Risk-based Assessment Does Not Distinguish Between Recent and Chronic HIV-1 Infection in Rio de Janeiro, Brazil
}

\begin{abstract}
Monica Merçon ${ }^{1}$, Suely H. Tuboi ${ }^{1}$, Sônia Maria Batista ${ }^{2}$, Sandra Regina Barros Telles ${ }^{1}$, José Roberto Grangeiro ${ }^{1}$, Roberto Zajdenverg $^{1}$, Regina Ferro do Lago ${ }^{1}$, Paulo Feijó Barroso ${ }^{3}$, Maria de Fátima Melo ${ }^{3}$ and Mauro Schechter ${ }^{3}$

${ }^{1}$ Projeto Praça Onze, Universidade Federal do Rio de Janeiro; ${ }^{2}$ Centro de Testagem e Aconselhamento, Hospital Escola São Francisco de Assis, Universidade Federal do Rio de Janeiro; ${ }^{3}$ Hospital Universitario Clementino Fraga Filho, Universidade Federal do Rio de Janeiro; Rio de Janeiro, RJ, Brazil
\end{abstract}

\begin{abstract}
This study investigated the risk factors associated with recent and chronic HIV infections among individual attending a voluntary counseling and testing (VCT) site in Rio de Janeiro, Brazil. In a cross-sectional study, recent HIV infections were detected by the sensitive/less-sensitive test, using Serologic Testing Algorithm for Recent HIV Seroconversion (STARHS) strategy, and compared to chronic HIV infection and HIV negative individuals. Seroincidence was estimated and risk factors associated with recent and chronic infections were assessed using multinomial logistic regression. Among the 7,379 individuals tested between June 2006 and April 2007, the overall prevalence and incidence of HIV infection were 7.5\%; and 1.39/100 PY, respectively. In multivariate analysis, having a HIV positive steady partner was a risk factor for recent and for chronic HIV infection for MSM, heterosexual male and women. No differences in risk factors for recent and chronic infections were found between MSM and heterosexual males. Among women, chronic infected individuals were more likely than HIV negatives to be older. Recently HIV infected women were more likely than HIV negatives to be less educated; and more likely than HIV negatives and chronically infected to report having more partners. Routinely used risk-based assessment in testing centers in Brazil lack sensitivity to distinguish between recent and chronic infections, particularly among MSM and heterosexual males. Steady relationships and serosorting may be playing a key role in maintaining the HIV epidemics in Brazil. Key-Words: STARHS, HIV seroconversion, VCT; Steady partner; HIV; HIV prevention.
\end{abstract}

Identification of individuals at high risk for HIV infection is of paramount importance for the conduct of studies on preventive strategies and on treatment of recent infections. The ability to differentiate recent from long-standing infection with the Serologic Testing Algorithm for Recent HIV Seroconversion (STARHS) [1] was a major contribution for estimating HIV-1 incidence, providing a simple tool for better understanding the dynamics of the HIV epidemic. STARHS, a two step testing strategy that consists of applying a less sensitive (LS) enzyme-linked immunoassay to a serologically confirmed case, relies on the fact that antibody levels are lower in early HIV infection than in established infections [1]. We conducted a cross sectional analysis to investigate risk factors associated with recent (RI) and chronic (CI) HIV infections using the STARHS strategy in a public voluntary counseling and testing (VCT) site in Rio de Janeiro, Brazil.

\section{Material and Methods}

The VCT site is located at Hospital Escola São Francisco de Assis of the Universidade Federal do Rio de Janeiro and

Received on 25 March 2009; revised 10 July 2009.

Address for correspondence: Dr. Monica Merçon, MD, MSc. Projeto Praça Onze. Hospital Escola São Francisco de Assis, Universidade Federal do Rio de Janeiro, Av. Presidente Vargas, 2863. Segundo andar - Cidade Nova Rio de Janeiro - RJ - Brazil - 20210.030. Phone: (55-21) 2273 9073, Fax: (55-21) 2273 8262, Email: monica.mercon@gmail.com. This work was partially supported by the Fogarty International Center, National Institutes of Health (3 D43 TW01038) for Dr Tuboi, and by the Conselho Nacional de Desenvolvimento Científico e Tecnológico (CNPq) for Dr. Schechter.

The Brazilian Journal of Infectious Diseases Publishing. All rights reserved. provides HIV counseling and testing to approximately 8,000 individuals per year. Data on demographic and risk behaviors are collected through a structured questionnaire that is used in all publicly funded VCT sites in Brazil. De-identified data are entered into a national database. As part of a study on recently acquired HIV-infection, all individuals who sought testing between June 2006 and April 2007 were offered a LS test in case their conventional serology was found to be positive. An informed consent for the performance of the LS assay (the BED Capture EIA [2]) was obtained at the initial visit, prior to collection of blood. Also in the initial visit, a questionnaire about demographics and risk behaviors was applied during the individual counseling. For the present report, de-identified demographic data, risk behaviors and previous diagnoses of sexually transmitted diseases (STDs) were abstracted from the VCT's database. Demographic variables included: sex, age and education (categorized as at least 8 years of formal education or less). Risk behaviors included: number and type of partners (steady versus casual) in the last 12 months; reported HIV-positive steady partner; condom use in the last sexual encounter with a casual partner; condom use with the steady partner; and STDs in the preceding 12 months. Barriers for not using condom with steady and casual partners were grouped into 5 categories: trust, condomrelated (cost/access, lack of knowledge, discomfort), inability to negotiate, carelessness, and other. Steady partnership was defined as a sexual relationship lasting at least 12 months; this definition allows more than one simultaneous steady partnership. The annual incidence estimates were obtained using the consensus formula

$$
\mathrm{I}=\frac{(365 / \mathrm{w}) \mathrm{N}_{\mathrm{inc}}}{\mathrm{N}_{\mathrm{neg}}+(365 / \mathrm{w}) \mathrm{N}_{\mathrm{inc}} / 2} \times 100
$$


and

$$
95 \% \mathrm{CI}=\mathrm{I} \pm 1.96 \frac{\mathrm{I}}{\left(\sqrt{\mathrm{N}_{\text {inc }}}\right)}
$$

where I = estimated incidence; $\mathrm{w}=$ window period; Ninc = number of recently infected; and Nneg = number of seronegative samples [3]. All analyses were stratified by risk category as men who have sex with men (MSM), heterosexual males, and females.

For univariate analyses, comparisons between RI, CI, and HIV Negative (HN) were made by Chi-square or Fisher's exact tests for categorical variables, and the Kruskal-Wallis test for continuous variables. To assess the relationship between risk factor and outcome, multinomial logistic regression models were fitted using $\mathrm{HN}$ as the reference group. Odds ratios and 95\% confidence intervals were calculated. Comparisons between RI and CI were made by the Wald test. Stata software (version 9.0; Stata) was used for the analyses.

\section{Results}

During the study period, 7,379 individuals were tested for HIV-1 infection. Of these, 851(11.5\%) were MSM, 2,718 (36.8\%) were heterosexual males, and 3,810 (51.6\%) were heterosexual females. The overall HIV-1 prevalence was 7.5\% $(553 / 7,379)$, and 47 subjects $(0.6 \%)$ were identified as RI. The overall estimated HIV seroincidence was 1.39 per 100 PY (95\%CI 1.19 - 1.59). HIV seroincidence was higher among MSM than heterosexual males and females [6.29 (95\%CI 4.87 - 7.69); vs 1.20 (95\%CI $0.88-1.50)$, and $0.66(0.47-0.85)$ per $100 \mathrm{PY}$, respectively, $\mathrm{p}<.01]$. The median number of partners in the preceding 12 months was 3 (IQR 1-6), 2 (IQR 1-4), and 1 (IQR 1-2) for MSM, male heterosexuals and female heterosexuals, respectively.

Among MSM, univariate analysis showed that HN, CI and RI did not differ statistically in terms of age [median 28 (IQR 24-38); 29 (24-36); and 31 (24-34) respectively; p=.79]; proportion of individuals who reported not having used condom in the last sexual encounter with a casual partner (72.2, 66.2, and 66.7\%; $\mathrm{p}=.54$ ); history of a STD in the preceding 12 months (10.6, 11.0, and $15.0 \%$; $\mathrm{p}=.82$ ); having had 4 or more partners in the preceding 12 months (36.6, 41.2, and 35.0; $\mathrm{p}=.38$ ); and having at least 8 years of formal education (87.7, 82.9, and 99.0\%, respectively; $\mathrm{p}=.06$ ). RI and CI were more likely than HN to report having a HIV-infected partner (20.0, 31.5 , and $17.4 \%$, respectively; $p=.01$ ). In all groups, the most frequently reported reason for inconsistent condom use with a steady partner was trust: $54.9,56.1$, and $44.4 \%$ for $\mathrm{HN}$, CI and RI, respectively. As for reasons for not using condoms with casual partners, carelessness was the most frequently reported reason by HN (29.6\%) and RIs (66.7\%), whereas condom-related issues were most frequently reported by CIs (27.6\%). In multivariate analysis adjusted for education and steady partner's risk, CI were more likely than HN to report having a HIV-infected partner.

Among heterosexual men, in univariate analysis, RI tended to be older than CI and HN [42 (31 - 50); 35 (29 - 44); and 33
( 25 - 44) respectively; $\mathrm{p}<.01]$. RI were more likely than CI and HN to have less than 8 years of formal education $(46.7,38.6$, and $30.4 \%$, respectively; $\mathrm{p}=.03$ ). As for risk behaviors, a similar proportion of HN, CI and RI reported not having used condom in the last sexual encounter with a casual partner (77.9, 77.4, and $66.7 \%$, respectively; $\mathrm{p}=.75$ ). With regards to the number of partners, no differences were found between the three groups: having had at least 4 sexual partners in the preceding 12 months was reported by $27.4,30.8$, and $26.7 \%$ of $\mathrm{HN}$, CI and RI, respectively $(p=.63)$. None of the RI reported a history of STD, a finding that did not differ statistically from CI and $\mathrm{HN}(0.0,11.4$ and $13.4 \%$, respectively; $\mathrm{p}=.23)$. The most common reason for not using condoms with the steady partner was trust (60.5, 50.5 and $75.0 \%$ for $\mathrm{HN}$, CI and RI, respectively). Condom-related issues were the most commonly reported reasons for not using condoms with casual partners in all groups (34.6, 30.2, and $60.0 \%$ for HN, CI and RI, respectively). Being HIV-positive was the most commonly reported partner's risk among RI (71.4\%) but not among CI and HN (30.9, and $8.8 \%$, respectively; $\mathrm{p}<.01$ ). In multivariate analysis controlled for age, education and partner's risk, RI and CI were more likely than HN to report having a HIV-positive partner.

Among women, univariate analyses showed that CI were older than HN and RI [37 (29 - 44); 30 (23 - 41); and 31 (26 44); $\mathrm{p}<.01]$. CI and RI reported fewer years of formal education than $\mathrm{HN}(50.7,50.0$, and $38.1 \%$ with $<8$ years; $p<.01)$. Rates of inconsistent condom use with casual partners were similar in all 3 groups (77.3, 69.2, and $100.0 \%$ for HN, CI and RI, respectively; $\mathrm{p}=.74$ ), as well as the proportion of women reporting a STD in the preceding 12 months $(8.8,11.5$, and $0.0 \%$ for $\mathrm{HN}, \mathrm{CI}$ and $\mathrm{RI} ; \mathrm{p}=.30$ ). The most commonly reported reason for not using condom with steady partners was trust (48.8, 51.8, and 55.6\% for HN, CI and RI); whereas no consistent pattern was reported for casual partners. Women in the RI group tended to report more sexual partners than those in the CI and HN groups (25.0, 8.1, and 7.2\%; $\mathrm{p}=.05$ ); and were more likely to report having a HIV-infected partner (50.0, 23.2, and $5.9 \% ; \mathrm{p}<.01$ ). Multivariate analysis controlled for age, education, number of partners and partner's risk showed that Recent HIV infected women were less educated than HN, more likely to report having had more partners, and to report having a HIV-infected partner. Results of multivariate analyses for all risk categories are shown in Table 1.

\section{Discussion}

We previously reported the estimated incidence of recent infections in men and in nonpregnant women using the STARHS algorithm [4,5]. The present results indicate that, on average, the incidence of RI in our setting has declined over time, a trend that remained even after excluding pregnant women from the analysis (data not shown). The higher estimated incidence of RI among MSM compared with heterosexual men and women suggests that the HIV transmission rate remains high within this risk category despite the extensive public health prevention efforts. 


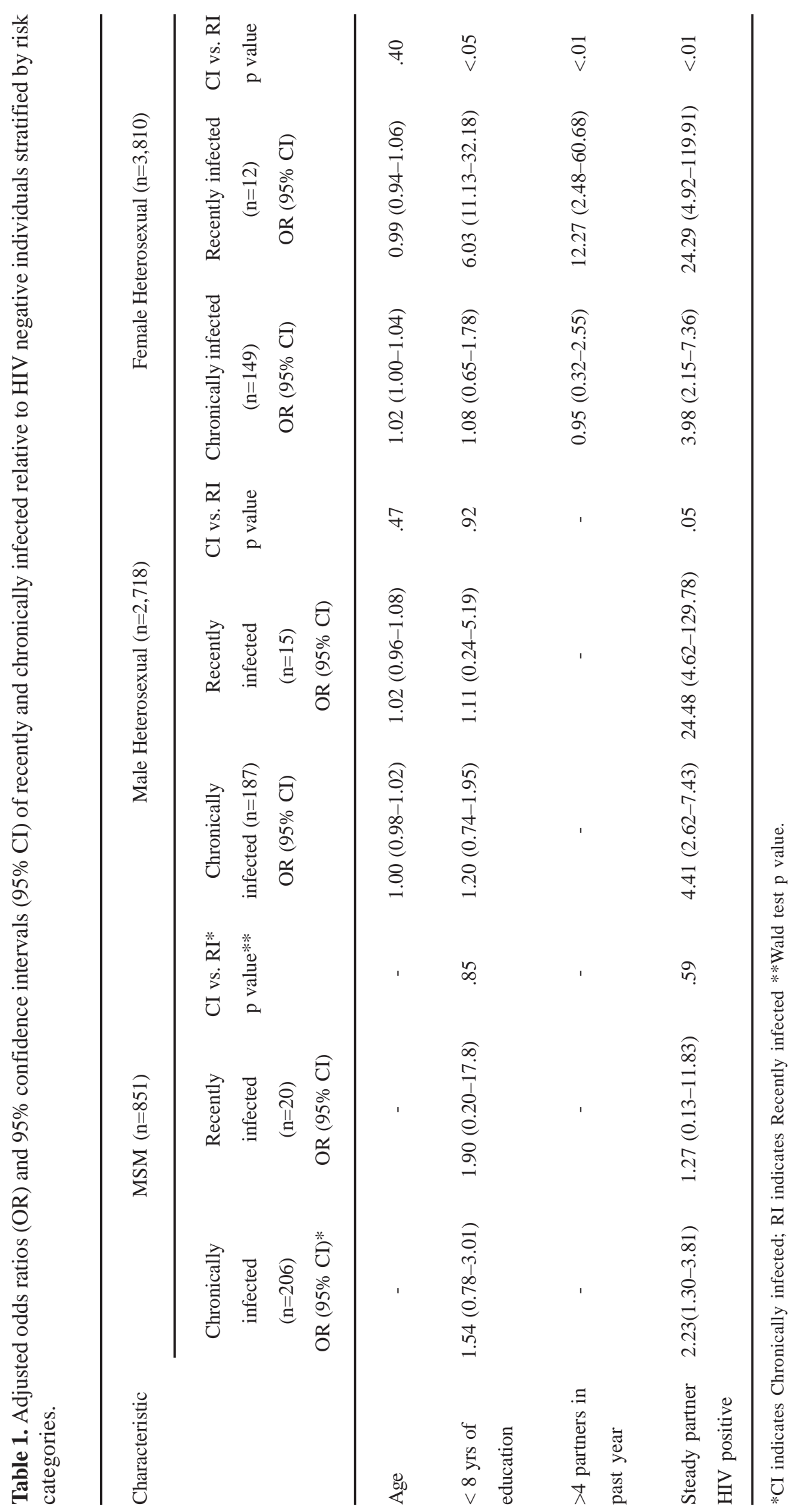


Our results suggest that the behavior-based risk assessment questionnaire that is being used in VCT sites in Brazil lacks sensitivity to distinguish RI from CIs, particularly among MSM and heterosexual men. No particular behavioral pattern was found to be associated with RI in the MSM risk category, when traditional confounders were taken into account. In a similar study conducted at a VCT site in Santos, Brazil, only a history of sex work was independently associated with risk of RI, whereas condom use, number of sexual partners, partner's HIV status and demographic characteristics did not differ between RI and CI [6]. Taken together, these results suggest that the risk assessment questionnaire that is currently used in VCT sites in Brazil may not effectively capture risk behaviors that are presently driving the HIV epidemic.

In a large study using the STAHRS algorithm in samples from 10 US cities, a previous history of urethral gonorrhea was independently associated with risk of RI [7]. In our study, no association was found between previous STD and risk of $\mathrm{RI}$ in all risk categories. In fact, individuals with RI reported fewer STDs in all risk categories. Given that having a HIVinfected steady partner was the strongest risk factor for RI, our results suggest that steady relationships may be playing an important role in the HIV epidemics in Brazil.

A trend toward older age of RI was seen among heterosexual men and women, when compared with CI and HN. This tendency was also reported in a population based study conducted in New York City, US [8], and may reflect testing practices among older versus younger men, whereby the former group seeks testing more frequently and are more likely to be diagnosed with RI. On the other hand, the association between RI and fewer years of formal education, a proxy for socio-economic status, in both heterosexual men and women categories is consistent with historical trends in Brazil, indicating that the epidemic continues to move to lower socio-economic strata of the population.

There are several limitations to our study, in particular the specificity of the BED-EIA, which has been reported to yield false positive results in AIDS cases and to overestimate incidence, particularly in settings with high prevalence of subtypes C and A1 [9]. Although we were not able to estimate the bias due to possible inclusion of individuals with AIDS, it is likely to be low, since the vast majority of individuals seeking testing are asymptomatic. Infecting subtypes are unlikely to have influenced our results, since subtypes $\mathrm{C}$ and $\mathrm{A}$ are rarely encountered in Rio de Janeiro, where subtypes are B and F represent almost the totality of infections $[10,11]$. The external validity of our results is also open to discussion, given that the study population does not necessarily represent the general population. Thus, the higher estimated incidence among MSM and older heterosexual males and females may be due to the fact that these populations may seek HIV testing more often. In addition, relatively few female sex workers currently seek testing at the Hospital Escola São Francisco de Assis VCT due to the availability of several outreach programs [12].
In conclusion, our study suggests that the structured questionnaire presently used in publicly funded VCT sites in Brazil lacks sensitivity to detect risk factors of recently HIV infected individuals, particularly MSM and heterosexual males. Additionally, our results suggest that steady relationships may be playing a key role in the HIV epidemics in Brazil. Further studies that address risk behaviors in detail are needed for better understanding the Brazilian epidemic and for guiding public health interventions.

\section{Acknowledgements}

The authors wish to thank all the patients, and collaborating Hospital São Fracisco de Assis - VCT staff, who made this study possible as well as Infectious Diseases Research Laboratory personnel for help and technical support.

\section{References}

1. Janssen RS, Satten GA, Stramer SL, et al. New testing strategy to detect early HIV-1 infection for use in incidence estimates and for clinical and prevention purposes. Jama 1998;280(1):42-8.

2. Parekh BS, Kennedy MS, Dobbs T, et al. Quantitative detection of increasing HIV type 1 antibodies after seroconversion: a simple assay for detecting recent HIV infection and estimating incidence. AIDS Res Hum Retroviruses 2002;18(4):295-307.

3. CDC. BED-CEIA Incidence and Adjustment Formula. Available at www.cdc.gov/nchstp/od/GAP/docs/surveillance/BEDCEIA\%20Incidence\%20and\%20Adjustment\%20Formula.pdf 2006.

4. Schechter M, do Lago RF, de Melo MF, et al. Identification of a high-risk heterosexual population for HIV prevention trials in Rio de Janeiro, Brazil. Projeto Praco Onze Study Group. J Acquir Immune Defic Syndr 2000;24(2):175-7.

5. Barroso PF, Harrison LH, de Fatima Melo M, et al. Identification of a high-risk heterosexual cohort for HIV vaccine efficacy trials in Rio de Janeiro, Brazil, using a sensitive/less-sensitive assay: an update. J Acquir Immune Defic Syndr 2004;36(3):880-1.

6. Alves K, Shafer KP, Caseiro M, et al. Risk factors for incident HIV infection among anonymous HIV testing site clients in Santos, Brazil: 1996-1999. J Acquir Immune Defic Syndr 2003;32(5):551-9.

7. Schwarcz S, Weinstock H, Louie B, et al. Characteristics of persons with recently acquired HIV infection: application of the serologic testing algorithm for recent HIV seroconversion in 10 US cities. J Acquir Immune Defic Syndr 2007;44(1):112-5.

8. Nash D, Bennani Y, Ramaswamy C, Torian L. Estimates of HIV incidence among persons testing for HIV using the sensitive/ less sensitive enzyme immunoassy, New York City, 2001. J Acquir Immune Defic Syndr 2005;39(1):102-11.

9. Karita E, Price M, Hunter E, et al. Investigating the utility of the HIV-1 BED capture enzyme immunoassay using cross-sectional and longitudinal seroconverter specimens from Africa. Aids 2007;21(4):403-8.

10. Morgado MG, Guimaraes ML, Gripp CB, et al. Molecular epidemiology of HIV-1 in Brazil: high prevalence of HIV-1 subtype B and identification of an HIV-1 subtype D infection in the city of Rio de Janeiro, Brazil. Evandro Chagas Hospital AIDS Clinical Research Group. J Acquir Immune Defic Syndr Hum Retrovirol 1998;18(5):488-94.

11. Bello G, Eyer-Silva WA, Couto-Fernandez JC, et al. Demographic history of HIV-1 subtypes B and F in Brazil. Infect Genet Evol 2007;7(2):263-70.

12. Barroso PF, de Souza MB, do Lago RF, et al. Barriers to Recruit Female Commercial Sex Workers for HIV Vaccine Trials: The Rio de Janeiro Experience. J Acquir Immune Defic Syndr 2009;50(1):116-117. 